\title{
¿Tiene género la ciencia? Conocimientos y actitudes hacia la Ciencia en niñas y niños de Educación Primaria
}

\author{
María Dapía Conde \\ Facultad de Ciencias de la Educación. Universidad de Vigo. Ourense. España. \\ dapia@uvigo.es \\ ORCID: https:// orcid.org/0000-0003-1348-2654
}

\author{
Ricardo Escudero Cid \\ Departamento de Química Física Aplicada. Universidad Autónoma de Madrid. España. \\ ricardo.escudero@uam.es \\ ORCID: https:// orcid.org/0000-0001-7708-3711
}

\author{
Manuel Vidal López \\ Facultad de Ciencias de la Educación. Universidad de Vigo. Ourense. España. \\ mvlopez@uvigo.es \\ ORCID: https:/ / orcid.org/0000-0001-9947-3745
}

[Recibido: 1 Febrero 2019. Revisado: 14 Abril 2019. Aceptado: 8 Julio 2019]

\begin{abstract}
Resumen: La menor presencia del género femenino en las profesiones científicas y tecnológicas sigue siendo una preocupación actual. Esta investigación pretende identificar los conocimientos y actitudes del alumnado de educación primaria sobre las aportaciones de la ciencia y los/as científicos y sus proyecciones profesionales en ser científicos/as, analizadas desde una perspectiva de género. Hemos aplicado un cuestionario a 378 estudiantes de educación primaria que posteriormente se han analizado con el paquete estadístico SPSS. Los resultados indican un conocimiento medio de las aportaciones de la ciencia y de los científico/as tanto en niñas como en niños y una alta coincidencia en las visiones expresadas, estableciéndose escasas asociaciones entre conocimientos y género. En las actitudes se constata una visión positiva, aunque asoman algunas diferencias entre niñas y niños, siendo estos últimos los que dan mayor confianza a la ciencia. Asimismo, en cuanto a su deseo de ser científico o científica tampoco se constata sesgo de género.
\end{abstract}

Palabras clave: Ciencia y tecnología; Enseñanza primaria; Conocimiento; Diferencias de género.

Does science have a gender? Knowledge and attitudes toward the Science of girls and boys in Primary Education

Abstract: Nowadays, an important concern is the lower presence of the female gender in scientific and technological professions. This work tries to identify the knowledge and attitudes of the students of primary education on the contributions of the science and the scientists. Although, this study aims to know their professional projections in science, analyzed from a gender perspective. It was carried out a test to a sample of 378 students of primary education which have subsequently been analyzed with the SPSS statistical package. The obtained results indicate an average knowledge of the contributions of science and scientists in both girls and boys, establishing few associations between knowledge of science and gender. Students show a positive vision about science, although some differences appear between girls and boys, e.g. males give greater confidence to science. Likewise, in terms of their desire to be scientific or scientific, there is no gender gap.

Keywords: Science and Technology; Primary education; Gender difference

Para citar este artículo: Dapía, M., Escudero-Cid, R., Vidal, M. (2019) ¿Tiene género la ciencia? Conocimientos y actitudes hacia la Ciencia en niñas y niños de Educación Primaria. Revista Eureka sobre Enseñanza y Divulgación de las Ciencias 16(3), 3201. doi: 10.25267/Rev_Eureka_ensen_divulg_cienc.2019.v16.i3.3302 


\section{Introducción}

Las actitudes y los conocimientos que tienen los estudiantes de las aportaciones de la ciencia y de los científicos/as son temas de gran actualidad; su interés se justifica en el continuo descenso observado en el número de estudiantes que prosiguen con estudios científicos (Archer et al. 2010, Fernández, Torío y García 2015, Gil-Flores 2012, Hill, Corbett y Rose 2010, Tytler y Osborne 2012), el papel fundamental que desempeña la ciencia y la tecnología en el desarrollo económico de cada país (Gago et al. 2004) y la importancia de la alfabetización científica entre la ciudadanía (OECD 2013) como posible contribución a la resolución de los problemas con que se enfrenta la humanidad en la actualidad (Gil-Pérez y Vilches 2006). Este preocupante panorama constituye un gran reto internacional, con investigaciones recientes sobre la influencia de determinados factores, entre ellos el género, en la elección de los estudiantes en carreras científicas (Arandia, Zuza y Guisasola 2016, Chachashvili-Bolotin, Milner-Bolotin y Lissitsa 2016, Khanyane, Mokuku y Nthathakane 2016, Vázquez y Manassero 2015).

Desde una perspectiva de género se ha indicado que las mujeres están insuficientemente representadas en las profesiones científicas y tecnológicas (Arandia et al. 2016, Bamberger, 2014, Holmegaard, Madsen y Ulriksen 2012, Osborne, Simon y Collins 2003, Rossi y Barajas 2015, Watts 2014) motivado por la existencia de estereotipos de género relacionados con el aprendizaje de la ciencia y la tecnología (Hill, Corbett y Rose 2010, Nosek et al. 2009, Wang y Degol 2017), por diferencias de género en experiencias motivacionales vividas en la etapa de secundaria (Patall, Steingut, Freeman, Pituch y Vázquez 2018), por su elección durante su trayectoria escolar (Hill et al. 2010) y no por un menor rendimiento o habilidades de las niñas (Simpson, Che y Bridges 2016).

Una revisión sobre las actitudes de los estudiantes de primaria hacia la ciencia escolar (Kerr y Murphy 2012) recoge un número importante de estudios a nivel internacional focalizados en la edad y el género de los estudiantes, con efectos muy significativos en muchos de ellos en comparación con otras variables (e.g. Dawson 2000, Murphy y Beggs 2003, Murphy, Beggs, Carlisle y Greenwood 2004). No obstante, revisiones anteriores (Schibeci 1984) han informado de diferentes estudios a nivel de primaria donde no se ha registrado ningún efecto de género o efectos significativamente pequeños entre niños y niñas (e.g. Haladyna y Shaughnessy 1982, Selim y Shrigley 1983).

Jones, Howe y Rua (2000) en una investigación sobre la influencia de género en las experiencias, intereses y actitudes hacia la ciencia y los científicos/as con alumnos de sexto grado (11-12 años) han señalado la existencia de diferencias significativas entre niños y niñas, relacionadas con sus experiencias extracurriculares y la importancia de la ciencia para su futuro empleo. En cuanto a sus experiencias extracurriculares, las diferencias encontradas apoyan la suposición histórica de que los niños tienden a tener más experiencias en ciencias físicas y las niñas en ciencias biológicas, las cuales no dejan de ser expresiones de los valores y actitudes de la cultura general, que en su visión tradicional transmiten lo que es más apropiado para las niñas (hacer pan, coser, plantar semillas, ...) y los niños (jugar con motores, baterías, poleas, ...) y que sesgan sus valores y actitudes hacia la ciencia y la tecnología en la escuela. Respecto a la importancia de la ciencia para su futuro trabajo han indicado que más niños que niñas querían "dirigir a otras personas", "tener un trabajo fácil", "hacerse famosos", "inventar y hacer cosas nuevas" y "ganar mucho dinero", mientras que más niñas que niños querían "ayudar a otras personas".

En una investigación longitudinal sobre cómo evoluciona el interés de los estudiantes hacia la ciencia y las carreras científicas, Archer et al. (2010) exploraron las actitudes con estudiantes de 10 a 11 años. En su estudio han señalado que la adopción de una identidad científica puede ser 
indeseable para muchos estudiantes, llegando a ser particularmente problemática para las niñas, donde sus pares masculinos juegan un papel activo en su exclusión de la ciencia, argumentando que ellos son intelectualmente mejores, que los científicos/as que conocen son todos hombres y que las chicas no están de forma natural en la ciencia porque "la moda y la ciencia no se mezclan" (Archer et al., 2010, p. 635). Asimismo, el estudio de Christidou, Bonoti y Kontopoulou (2016) analizó la imagen de los estudiantes de primaria sobre científicos y científicas utilizando una adaptación del DAST (Draw-A-Scientist-Test); los resultados confirman el impacto de género siendo los niños los que utilizaban más indicadores estereotípicos que las niñas. Este punto de vista masculino puede afectar de manera negativa a la actitud de las niñas hacia la ciencia a lo largo de su formación obligatoria, desanimándolas a no proseguir estudios científicos.

En nuestro país, no se ha prestado demasiada atención al análisis específico de las actitudes hacia la ciencia y la tecnología en la Educación Primaria. Nortes y Pro (2010) concluyen, que la opinión sobre la ciencia de niños de 11-12 años es una visión positiva, no tan estereotipada como podría esperarse, con muy pocas diferencias significativas y de difícil interpretación entre niñas y niños, sorprendiendo el elevado porcentaje de niños que indica que la ciencia es una profesión masculina. Delgado, Vallés y López (2014), en un trabajo sobre las actitudes de los estudiantes de quinto y sexto de Primaria, han señalado también la valoración positiva del alumnado hacia el papel de la ciencia y los científicos/as en nuestra sociedad, mostrando una especial preocupación por la higiene y la salud y ciertas contradicciones o desconfianza hacia la tecnología y sus efectos en la preservación del medioambiente.

Varias investigaciones han estudiado las actitudes de los estudiantes de primaria y secundaria hacia diversos aspectos de la ciencia y la tecnología, de las que se pueden extraer de modo generalizado ciertas conclusiones de interés; como una valoración más positiva en los estudiantes de primaria acerca de los científicos/as y su trabajo (Pro, Tárraga y Pérez 2009), un deterioro de las actitudes relacionadas con la ciencia a medida que aumenta su edad (Vázquez y Manassero 2007) y un posicionamiento favorable ante dicotomías relacionadas con las aportaciones de la ciencia y los científicos/as (Pro y Pérez 2014). Otros estudios (Pérez 2012, Vázquez y Manassero 2011) que han analizado la evolución de las actitudes de los estudiantes hacia la ciencia desde los últimos cursos de primaria a secundaria (incluyendo la ciencia escolar, la visión de los científicos/as, la imagen y el interés vocacional de la ciencia y tecnología) indican una percepción positiva de los estudiantes de primaria hacia la ciencia y tecnología, aunque sin mostrar diferencias significativas en función del género; con un conocimiento medio-aceptable de niños y niñas sobre las aportaciones de la ciencia y los científicos/as (Pérez 2012), pero con una valoración positiva que va disminuyendo a medida que avanzan de cuarto a sexto de Primaria (Vázquez y Manassero 2011).

En este contexto, la presente investigación se ha centrado en identificar los conocimientos y actitudes del alumnado de educación primaria sobre las aportaciones de la ciencia y los/as científicos y sus proyecciones profesionales en ser científicos o científicas, desde una perspectiva de género.

\section{Metodología}

\section{Población y muestra}

La población en este estudio viene definida por el alumnado de $6^{\circ}$ de primaria matriculado en centros públicos y concertados de la provincia de Ourense, en el curso 2015-16. La muestra estuvo constituida por un total de 378 sujetos, de diferentes municipios seleccionados mediante un procedimiento de muestreo multietápico estratificado por ámbito -rural y urbano- y por titularidad de centro -público (CEIP) y privado (CPR), con un error muestral 
de un 5\%. En total participaron 13 centros -6 urbanos y 7 rurales- y 19 aulas diferentes de los distintos centros.

La edad media de la muestra participante es de 11.44 años $(\mathrm{S} x=0.599)$, en un rango de 11 a 13 años. En función del género, el 52.9\% son niños y el $47.1 \%$ son niñas. Por ámbito geográfico el $55.6 \%$ de los estudiantes corresponde al urbano y el $44.4 \%$ al rural.

\section{Instrumento}

Para la recogida de datos se elaboró un cuestionario que contiene, además de los datos sociodemográficos, diferentes escalas extraídas de cuestionarios relacionados con la imagen que tienen los estudiantes de las ciencias. El primero de ellos se denomina PANA (Proyecto de Actitudes hacia las ciencias en Niños y Adolescentes, Pérez y Pro 2005) y de él se obtuvieron los ítems referidos a las actitudes ante la ciencia y los científicos/as, y otro bloque relativo a conocimientos sobre las aportaciones de la ciencia y los científicos/as. En segundo lugar, hemos utilizado uno de los apartados/escalas del cuestionario ROSE (Schreiner y Sjøberg 2004), en concreto hemos aplicado una escala Likert de cuatro puntos ("Muy en desacuerdo", "En desacuerdo", "De acuerdo" y "Muy de acuerdo") con 16 afirmaciones titulada Mis opiniones sobre ciencia y tecnología. Para esta última escala, en nuestro estudio, el índice de fiabilidad calculado por el estadístico alfa de Cronbach es de 0.75 y para la escala de actitudes extraída de PANA es de 0.64. Los instrumentos empleados se recogen en el anexo 1.

\section{Procedimiento de recogida y análisis de datos}

Para iniciar el proceso de recogida de datos, se contactó con los directores de los centros, para solicitar su autorización y acordar fecha para la administración de los cuestionarios. Al tratarse de menores desde los centros se ha enviado autorización a los padres y/o tutores para que manifestaran su consentimiento.

Tanto los responsables de los centros, como profesores y menores implicados fueron informados de la finalidad del estudio; estos últimos fueron invitados a participar de forma anónima y voluntaria, previa autorización de padres y/o tutores, garantizándoles la confidencialidad de la información y la protección de los datos. La tasa de rechazo se situó en el $1 \%$ de la población.

A cada participante se le entregó un único documento que contenía los instrumentos de medida descritos y se les solicitaba la mayor sinceridad posible y a todos los participantes se les facilitó la misma batería de instrumentos. La aplicación se realizó de forma colectiva en las respectivas aulas, entre los meses de febrero a mayo de 2016 por la misma investigadora, a fin de evitar un factor de sesgo.

Una vez los cuestionarios estaban cumplimentados se codificaban y se introducían en el programa estadístico SPSS, en su decimonovena versión para Windows v. 22.0, utilizado para su análisis. Como variable dependiente para el análisis se emplearon las propias medidas de los cuestionarios presentados y como variable independiente se usó el género (mujer/ hombre).

Para analizar los datos obtenidos se hizo uso de técnicas de análisis descriptiva univariada recurriendo a estadísticos de tendencia central y dispersión (medias y desviaciones típicas), análisis, frecuencias, porcentajes; los contrastes analíticos se ejecutaron mediante chi cuadrado y tras la verificación del supuesto de normalidad, mediante el método algebraico de Kolmogorov Smirnov, para las diferencias de medias se aplicó el estadístico no paramétrico Mann-Whitney-U (para muestras independientes). En todos los análisis el nivel de significación se fijó en $\mathrm{p}<.05$. 


\section{Resultados}

\section{Conocimientos sobre la ciencia: aportaciones de la ciencia y de los científicos/as}

Para aproximarnos a los conocimientos de niños y niñas se emplearon dos preguntas del cuestionario. En el primero, se ofrece un listado de diferentes conceptos y se les solicita que elijan, según la consideración de que puedan ser atribuidos fundamentalmente a los científicos/as ("Si’), con un "No", cuando consideran que no se deben a ellos, "En parte" si los y las científicos han participado, pero no han sido los únicos y "No lo se", si la respuesta no se está clara. En los 8 conceptos presentados solo se admitía como correcta una única opción. La tabla 1 recoge los resultados obtenidos diferenciando entre niños y niñas, atendiendo al porcentaje de respuestas correctas, incorrectas y no contestadas.

En primer lugar, llama la atención el elevado porcentaje de respuestas incorrectas existentes, particularmente en algunos de los objetos (de uso cotidiano); por ejemplo, en el ítem relativo al pan, el porcentaje de respuesta incorrecta en las niñas alcanza el 73.6\% y sólo un escaso $10 \%$ considera que el pan es una de las aportaciones de la ciencia. Por el contrario, la mayoría tienen claro (porcentajes de error rondan el 20\%) que las bombas o los teléfonos se los debemos a la ciencia. En una posición intermedia, bastante elevada, se sitúan la rueda y bicicleta que, para más del $40 \%$ no son considerados productos derivados de la actividad científica.

Tabla 1. Descubrimiento de la ciencia (frecuencias y porcentajes por género).

\begin{tabular}{|c|c|c|c|c|c|c|}
\hline & Respuestas & Total N (\%) & Niños n $(\%)$ & Niñas n $(\%)$ & $\mathrm{X}^{2}$ & Sig \\
\hline Cuadro de un pintor & $\begin{array}{l}\text { Correcta } \\
\text { Incorrecta } \\
\text { NS/NC }\end{array}$ & $\begin{array}{c}280(74.3) \\
68(18.0) \\
29(7.7)\end{array}$ & $\begin{array}{c}138(69.3) \\
46(23.1) \\
15(7.5)\end{array}$ & $\begin{array}{c}142(79.8) \\
22(12.4) \\
14(7.9)\end{array}$ & 7.41 & .025 \\
\hline Árboles & $\begin{array}{l}\text { Correcta } \\
\text { Incorrecta } \\
\text { NS/NC }\end{array}$ & $\begin{array}{c}228(60.3) \\
118(31.2) \\
32(8.5)\end{array}$ & $\begin{array}{c}116(58.0) \\
65(32.5) \\
19(9.5)\end{array}$ & $\begin{array}{c}112(62.9) \\
53(29.8) \\
13(7.3)\end{array}$ & 1.14 & .566 \\
\hline Rueda & $\begin{array}{l}\text { Correcta } \\
\text { Incorrecta } \\
\mathrm{NS} / \mathrm{NC}\end{array}$ & $\begin{array}{c}157(41.8) \\
167(44.4) \\
52(13.8)\end{array}$ & $\begin{array}{c}90(45.0) \\
91(45.5) \\
19(9.5)\end{array}$ & $\begin{array}{l}67(38.1) \\
76(43.2) \\
33(18.8)\end{array}$ & 6.98 & .030 \\
\hline Pan & $\begin{array}{l}\text { Correcta } \\
\text { Incorrecta } \\
\mathrm{NS} / \mathrm{NC}\end{array}$ & $\begin{array}{c}49(13.0) \\
270(71.6) \\
58(15.4)\end{array}$ & $\begin{array}{c}30(15.1) \\
139(69.8) \\
30(15.1)\end{array}$ & $\begin{array}{c}19(10.7) \\
131(73.6) \\
28(15.7)\end{array}$ & 1.61 & .447 \\
\hline Moda & $\begin{array}{l}\text { Correcta } \\
\text { Incorrecta } \\
\mathrm{NS} / \mathrm{NC}\end{array}$ & $\begin{array}{c}238(63.3) \\
81(21.5) \\
57(15.2)\end{array}$ & $\begin{array}{c}123(61.5) \\
45(22.5) \\
32(16.0)\end{array}$ & $\begin{array}{l}115(65.3) \\
36(20 ., 5) \\
25(14.2)\end{array}$ & 0.60 & .741 \\
\hline Teléfonos & $\begin{array}{l}\text { Correcta } \\
\text { Incorrecta } \\
\mathrm{NS} / \mathrm{NC}\end{array}$ & $\begin{array}{c}282(74.8) \\
72(19.1) \\
23(6.1)\end{array}$ & $\begin{array}{c}146(73.0) \\
44(22.0) \\
10(5.0)\end{array}$ & $\begin{array}{c}136(76.8) \\
28(15.8) \\
13(7.3)\end{array}$ & 2.91 & .234 \\
\hline Bomba & $\begin{array}{l}\text { Correcta } \\
\text { Incorrecta } \\
\mathrm{NS} / \mathrm{NC}\end{array}$ & $\begin{array}{c}239(63.4) \\
88(23.3) \\
50(13.3)\end{array}$ & $\begin{array}{c}135(67.8) \\
38(19.1) \\
26(13.1)\end{array}$ & $\begin{array}{c}104(58.4) \\
50(28.1) \\
24(13.5)\end{array}$ & 4.59 & .101 \\
\hline Bicicletas & $\begin{array}{l}\text { Correcta } \\
\text { Incorrecta } \\
\mathrm{NS} / \mathrm{NC}\end{array}$ & $\begin{array}{c}130(34.7) \\
181(48.5) \\
63(16.8)\end{array}$ & $\begin{array}{c}57(29.1) \\
102(52.0) \\
37(18.9)\end{array}$ & $\begin{array}{l}73(41.0) \\
79(44.4) \\
26(14.6)\end{array}$ & 5.97 & .500 \\
\hline
\end{tabular}

En segundo lugar, tal como se aprecia en la misma tabla, no puede hablarse de una alta asociación entre los conocimientos de la ciencia y el género; en la prueba chi cuadrado se manifiesta únicamente en dos ítems: cuadro de un pintor $\left(\mathrm{X}^{2}=7.41 ; \mathrm{p}=.025\right)$ y rueda $\left(\mathrm{X}^{2}=\right.$ 
6.98; $\mathrm{p}=.030$ ), siendo las niñas las que presentan mayores conocimientos en el primero y los niños en la rueda.

En la segunda pregunta, se presentaban 12 eventos u objetos y se les requería a los estudiantes que indicasen si creían que se debían a las y los científicos o no, ante cuatro posibles alternativas de respuesta ("Sï" "No", "Sípero solo en parte" o "No lo sê"). En algunos ítems había una opción de respuesta correcta mientras que en dos (Revolución Industrial y huevo frito) se admitían como válidas "Sî" y "Sí, pero solo en parte".

Como puede apreciarse en la figura 1, el grado de conocimiento de las aportaciones de los científicos/as varía para cada una de ellas, manifestándose importantes diferencias (ver porcentajes de respuestas correctas). Los estudiantes, de forma mayoritaria, identifican las vacunas o los viajes espaciales como contribuciones de las y los científicos; asimismo reconocen que, en las reglas de fútbol, en el Quijote y en la Constitución española no hay intervención de los científicos/as. Por el contrario, desconocen mayoritariamente la participación hecha por los científicos/as en los coches de Fórmula 1, el huevo frito o el fuego. Destacar, por último, que sólo el 39.4\% considera independiente la ciencia y la predicción de futuro que hacen los horóscopos.

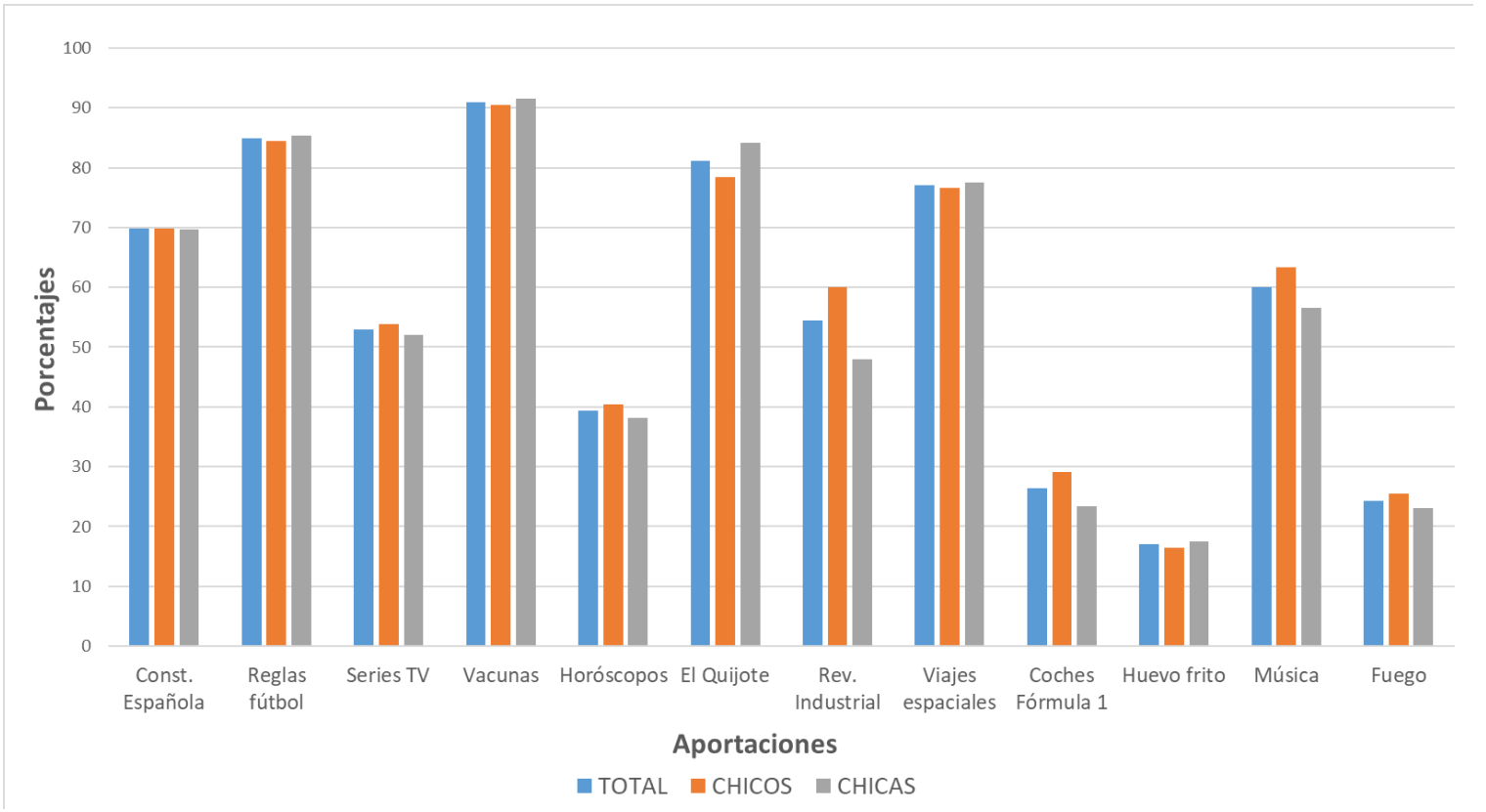

Figura 1. Aportaciones de los/as científicos/as (porcentaje de respuestas correctas por género).

Por otro lado, se confirma la alta coincidencia en las visiones sobre las contribuciones de los científicos/as entre niños y niñas; únicamente se constata asociación, prueba chi cuadrado, en relación con el Quijote $\left(\mathrm{X}^{2}=6.69 ; \mathrm{p}=.035\right)$ en favor de ellas y con el fuego $\left(\mathrm{X}^{2}=6.18 ; \mathrm{p}=.046\right)$ de ellos; en el resto de las aportaciones hay sensibles diferencias que no alcanzan significación estadística.

\section{Valoración de la ciencia}

La tabla 2 recoge las valoraciones que hacen los estudiantes ante una serie de afirmaciones relativas a la ciencia y ante las cuales ellos debían situarse eligiendo entre Nada de acuerdo (1), Poco de acuerdo (2), Bastante de acuerdo (3) y Totalmente de acuerdo (4). Como puede apreciarse, en general, hay una valoración aceptable en las opiniones vertidas; tanto chicas como chicos expresan opiniones bastante positivas ante la ciencia, con medias superiores a 3, en curiosidad, influencia positiva en el futuro y utilidad. Asimismo, en las proposiciones de connotación 
negativa, las medias son inferiores a 2 (Nada-Poco de acuerdo), con la única excepción de la proposición "la ciencia nos defiende de los que tratan de engañarnos", en la que la inclinación es menos acusada.

Tabla 2. Opiniones ciencia (media, desviaciones y rangos promedio por género).

\begin{tabular}{|c|c|c|c|c|}
\hline & $\begin{array}{c}\text { Niños } \\
\text { Media (Sx) } \\
\text { Rango Promedio }\end{array}$ & $\begin{array}{c}\text { Niñas } \\
\text { Media }(\mathrm{Sx}) \\
\text { Rango Promedio }\end{array}$ & $\begin{array}{c}\text { Mann- } \\
\text { Whitney } \\
\text { U }\end{array}$ & Sig \\
\hline La curiosidad es esencial en la ciencia & $\begin{array}{l}3.45(0.73) \\
191.45\end{array}$ & $\begin{array}{c}3.40(0.78) \\
188.26\end{array}$ & 17224.00 & .603 \\
\hline La ciencia debe ser eliminada de la escuela & $\begin{array}{l}1.17(0.53) \\
189.28\end{array}$ & $\begin{array}{l}1.16(0.46) \\
189.74\end{array}$ & 17756.50 & .943 \\
\hline $\begin{array}{l}\text { La ciencia no tiene mucho interés para la gente } \\
\text { que no es científica }\end{array}$ & $\begin{array}{l}1.88(0.93) \\
192.42\end{array}$ & $\begin{array}{l}1.77(0.80) \\
184.09\end{array}$ & 16831.50 & .424 \\
\hline Un futuro mejor depende de la ciencia & $\begin{array}{l}3.31(0.79) \\
199.22\end{array}$ & $\begin{array}{l}3.12(0.86) \\
176.44\end{array}$ & 15477.50 & .029 \\
\hline La ciencia es completamente aburrida & $\begin{array}{l}1.37(0.69) \\
188.79\end{array}$ & $\begin{array}{l}1.38(0.70) \\
187.13\end{array}$ & 17377.50 & .852 \\
\hline La ciencia es tremendamente útil & $\begin{array}{l}3.38(0.94) \\
190.84\end{array}$ & $\begin{array}{l}3.35(0.89) \\
182.75\end{array}$ & 16594.00 & .412 \\
\hline $\begin{array}{l}\text { La ciencia nos defiende de los que tratan de } \\
\text { engañarnos }\end{array}$ & $\begin{array}{l}2.44(1.17) \\
205.10\end{array}$ & $\begin{array}{l}2.08(1.11) \\
171.98\end{array}$ & 14681.00 & .002 \\
\hline
\end{tabular}

Por otro lado, destacaremos que esta imagen es bastante similar entre chicos y chicas, detectándose diferencias estadísticamente significativas en las afirmaciones "un futuro mejor depende de la ciencia" y "la ciencia nos defiende de los que tratan de engañarnos"; en ambas consideraciones la media es superior para los chicos, lo que implica que éstos presentan una valoración positiva mayor o más confianza en la ciencia. En otro apartado se preguntaba acerca de las aportaciones que, para ellos, había hecho la ciencia, consideradas globalmente. Las respuestas aparecen recogidas en la tabla 3 , en la que se puede apreciar una valoración altamente positiva en chicas y chicos; con un claro predominio de las aportaciones positivas -en ambos grupos la suma de los que consideran que la ciencia solo hizo aportaciones positivas y de los que creen que hizo más positivas que negativas supera el 70\%-. No obstante, cabe destacar que más de un $20 \%$ no se pronuncia, manifestando su desconocimiento al elegir la categoría "No sé", lo que parece indicar falta de posicionamiento claro.

Por otro lado, la prueba chi cuadrado, constata que hay asociación entre la valoración global que hacen los estudiantes de primaria y el género; una actitud más optimista es expresada por los niños.

Tabla 3. Opinión aportaciones ciencia (frecuencias y porcentajes por género).

\begin{tabular}{lccccc}
\hline & Total $\mathrm{N}(\%)$ & Niños $\mathrm{n}(\%)$ & Niñas $\mathrm{n}(\%)$ & $\mathrm{X}^{2}$ & Sig \\
\hline Sólo aportaciones positivas & $39(10.4)$ & $29(14.6 \%)$ & $10(5.7)$ & 10.56 & .032 \\
Más positivas que negativas & $227(60.7)$ & $110(55.6)$ & $117(66.5)$ & & \\
Más negativas que positivas & $25(6.7)$ & $16(8.1)$ & $9(5.1)$ & & \\
Sólo aportaciones negativas & $3(0.8)$ & $2(1.0)$ & $1(0.6)$ & & \\
No sé & $80(21.4)$ & $41(20.7)$ & $39(22.2)$ & & \\
\hline
\end{tabular}

La opinión sobre la ciencia se completa con la manifestada en la escala Mis opiniones sobre ciencia y tecnología cuyos resultados figuran en la tabla 4. La media en la escala es de 2.85 sobre 4, reflejando, por tanto, una opinión discreta sobre la ciencia y la tecnología. Las valoraciones más positivas, con medias superiores a 3 , se obtienen en los beneficios de la ciencia y la tecnología para la sociedad, el tratamiento de las enfermedades, las mejoras para las 
generaciones futuras, hacer la vida más saludable, fácil y cómoda, así como en el desarrollo de los países. Asimismo, hay una clara uniformidad en las opiniones de niños y niñas, no encontrándose apenas diferencias estadísticamente significativas, salvo en tres de las dieciséis cuestiones; los niños ven con mayor claridad que los beneficios de la ciencia son más importantes que los perjuicios que causan, así como que la ciencia y la tecnología pueden resolver casi todos los problemas, confirmándose, por tanto, mayor optimismo y confianza en el género masculino.

Tabla 4. Escala final "Mis opiniones sobre ciencia y tecnología" (Medias, desviaciones y rangos promedio según género).

\begin{tabular}{|c|c|c|c|c|}
\hline Ítems & $\begin{array}{c}\text { Niños } \\
\text { Media }(\mathrm{Sx}) \\
\text { Rango Promedio }\end{array}$ & $\begin{array}{c}\text { Niñas } \\
\text { Media }(\mathrm{Sx}) \\
\text { Rango Promedio }\end{array}$ & $\begin{array}{c}\text { Mann- } \\
\text { Whitney } \\
\text { U }\end{array}$ & Sig \\
\hline $\begin{array}{l}\text { 1. La ciencia y la tecnología son importantes para la } \\
\text { sociedad }\end{array}$ & $\begin{array}{c}3.36(0.65) \\
183.86\end{array}$ & $\begin{array}{c}3.41(0.61) \\
191.59\end{array}$ & 16703.50 & .437 \\
\hline $\begin{array}{l}\text { 2. La ciencia y la tecnología encontraron solución a } \\
\text { enfermedades como VIH/SIDA, cáncer,... }\end{array}$ & $\begin{array}{c}3.44(0.79) \\
184.98\end{array}$ & $\begin{array}{l}3.46(0.68) \\
183.96\end{array}$ & 16784.00 & .917 \\
\hline $\begin{array}{l}\text { 3. Gracias a la ciencia y la tecnología habrá más } \\
\text { oportunidades para las generaciones futuras. }\end{array}$ & $\begin{array}{l}3.14(0.76) \\
179.37\end{array}$ & $\begin{array}{c}3.23(0.72) \\
191.38\end{array}$ & 15850.00 & .231 \\
\hline $\begin{array}{l}\text { 4. La ciencia y la tecnología hacen nuestra vida más } \\
\text { saludable fácil y cómoda. }\end{array}$ & $\begin{array}{l}3.15(0.83) \\
186.13\end{array}$ & $\begin{array}{l}3.11(0.73) \\
178.46\end{array}$ & 15823.00 & .448 \\
\hline $\begin{array}{l}\text { 5. Las nuevas tecnologías hacen el trabajo más } \\
\text { interesante. }\end{array}$ & $\begin{array}{l}2.80(0.92) \\
183.93\end{array}$ & $\begin{array}{l}2.79(0.84) \\
184.08\end{array}$ & 16756.00 & .988 \\
\hline $\begin{array}{l}\text { 6. Los beneficios de la ciencia son más importantes } \\
\text { que los perjuicios que causan. }\end{array}$ & $\begin{array}{l}2.68(0.82) \\
182.73\end{array}$ & $\begin{array}{l}2.48(0.78) \\
160.93\end{array}$ & 12848.00 & .300 \\
\hline $\begin{array}{l}\text { 7. La ciencia y la tecnología ayudan a erradicar la } \\
\text { pobreza y el hambre en el mundo. }\end{array}$ & $\begin{array}{l}2.35(0.94) \\
179.56\end{array}$ & $\begin{array}{c}2.28(0.89) \\
172.87\end{array}$ & 14791.50 & .518 \\
\hline $\begin{array}{l}\text { 8. La ciencia y la tecnología pueden resolver casi } \\
\text { todos los problemas. }\end{array}$ & $\begin{array}{c}2.66(0.83) \\
193.95\end{array}$ & $\begin{array}{l}2.47(0.75) \\
171.11\end{array}$ & 14544.00 & .026 \\
\hline 9. La ciencia y la tecnología ayudan a los pobres. & $\begin{array}{l}2.17(0.91) \\
190.76\end{array}$ & $\begin{array}{l}2.00(0.78) \\
174.38\end{array}$ & 15117.50 & .111 \\
\hline $\begin{array}{l}\text { 10. La ciencia y la tecnología son la causa de los } \\
\text { problemas ambientales. }\end{array}$ & $\begin{array}{l}2.52(0.95) \\
185.55\end{array}$ & $\begin{array}{l}2.64(0.83) \\
172.42\end{array}$ & 14754.50 & .207 \\
\hline $\begin{array}{l}\text { 11. Un país desarrollado necesita de la ciencia y la } \\
\text { tecnología. }\end{array}$ & $\begin{array}{l}3.14(0.79) \\
195.18\end{array}$ & $\begin{array}{c}3.01(0.79) \\
177.84\end{array}$ & 15724.00 & .092 \\
\hline $\begin{array}{l}\text { 12. La ciencia y la tecnología benefician } \\
\text { principalmente a los países desarrollados. }\end{array}$ & $\begin{array}{c}2.98(0.87) \\
175.51\end{array}$ & $\begin{array}{l}2.89(0.78) \\
188.50\end{array}$ & 15114.00 & .207 \\
\hline $\begin{array}{l}\text { 13. Los científicos/as siguen el método científico, que } \\
\text { los lleva a respuestas correctas. }\end{array}$ & $\begin{array}{l}2.94(0.81) \\
173.36\end{array}$ & $\begin{array}{l}2.93(0.70) \\
168.33\end{array}$ & 14053.00 & .603 \\
\hline $\begin{array}{l}\text { 14. Hemos de confiar siempre en lo que dicen los } \\
\text { científicos y las científicas. }\end{array}$ & $\begin{array}{c}2.40(0.86) \\
183.19\end{array}$ & $\begin{array}{c}2.33(0.82) \\
176.29\end{array}$ & 15403.00 & .501 \\
\hline 15. Las personas científicas son neutrales y objetivas. & $\begin{array}{c}2,79(0.87) \\
153.66\end{array}$ & $\begin{array}{c}2.83(0.76) \\
157.86\end{array}$ & 11511.00 & .658 \\
\hline $\begin{array}{l}\text { 16. Las teorías científicas se desarrollan y cambian } \\
\text { constantemente. }\end{array}$ & $\begin{array}{l}3.02(0.88) \\
186.62\end{array}$ & $\begin{array}{l}2.87(0.76) \\
165.92\end{array}$ & 13680.50 & .039 \\
\hline Puntuación total & $\begin{array}{c}2.87(0.39) \\
118.91\end{array}$ & $\begin{array}{l}2.83(0.34) \\
115.62\end{array}$ & 6511.50 & .712 \\
\hline
\end{tabular}

\section{Los científico/as: atribuciones e intereses}

En una escala de 1-4 (Nada de acuerdo-Totalmente de acuerdo), si nos detenemos en las medias obtenidas (Ver tabla 5), la imagen que los estudiantes tienen de los científicos/as vendría definida fundamentalmente por personas que se entusiasman con lo que hacen, que su trabajo beneficia a todos, hacen un trabajo divertido y ganan dinero. En menor medida, son considerados personas con muchos amigos, que trabajan todo el día, que son divertidas y que nadie les discute por la precisión de sus descubrimientos. Asimismo, consideran que no son 
características propias de las y los científicos ser personas despistadas, famosas o del género masculino.

Aunque éste es el perfil general que dibujan los estudiantes, se constatan diferencias estadísticamente significativas entre niños y niñas en las siguientes atribuciones: sólo trabajan en países ricos, se preocupa más de sus investigaciones que de los problemas "normales" de la sociedad, ser una persona despistada, ser famoso y trabajar todo el día, siendo los chicos los que presentan una valoración más alta en todas las caracterizaciones salvo en que trabaja todo el día.

Tabla 5. Los científicos/as vistos por niñas y niños (medias y desviaciones típicas por género).

\begin{tabular}{lccccc}
\hline & $\begin{array}{c}\text { Total } \\
\text { Media }\left(\mathrm{S}_{\mathrm{x}}\right)\end{array}$ & $\begin{array}{c}\text { Niños } \\
\text { Media }\left(\mathrm{S}_{\mathrm{x}}\right)\end{array}$ & $\begin{array}{c}\text { Niñas } \\
\text { Media }\left(\mathrm{S}_{\mathrm{x}}\right)\end{array}$ & F & Sig \\
\hline Gana dinero & $2.96(0.76)$ & $2.94(0.81)$ & $2.99(0.70)$ & 0.40 & .526 \\
Trabajo divertido & $3.04(0.90)$ & $3.09(0.88)$ & $2.98(0.91)$ & 1.37 & .243 \\
Entusiasmo & $3.38(0.69)$ & $3.36(0.70)$ & $3.40(0.69)$ & 0.26 & .608 \\
Beneficia a todos & $3.36(0.81)$ & $3.39(0.76)$ & $3.32(0.85)$ & 0.67 & .412 \\
Muchos amigos & $2.66(0.85)$ & $2.73(0.81)$ & $2.58(0.88)$ & 3.10 & .079 \\
Sólo habla de ciencias & $1.50(0.83)$ & $1.57(0.90)$ & $1.41(0.73)$ & 3.26 & .072 \\
Trabaja países ricos & $1.42(0.74)$ & $1.46(0.79)$ & $1.34(0.65)$ & 3.92 & .049 \\
No se preocupa de los problemas sociedad & $1.81(0.85)$ & $1.96(0.92)$ & $1.63(0.73)$ & 14.07 & .000 \\
Despistado & $1.31(0.59)$ & $1.38(0.68)$ & $1.24(0.45)$ & 5.31 & .022 \\
Famoso & $1.22(0.52)$ & $1.31(0.62)$ & $1.12(0.38)$ & 12.14 & .001 \\
Es de hombres & $1.20(0.59)$ & $1.25(0.69)$ & $1.14(0.45)$ & 3.02 & .083 \\
Trabaja todo el día & $2.64(0.86)$ & $2.37(0.87)$ & $2.53(0.84)$ & 5.09 & .025 \\
Persona divertida & $2.58(0.88)$ & $2.66(0.90)$ & $2.50(0.84)$ & 2.93 & .088 \\
Investigan a su antojo & $1.59(0.88)$ & $1.67(0.94)$ & $1.50(0.79)$ & 3.56 & .060 \\
Nadie les discute & $2.21(0.98)$ & $2.30(1.00)$ & $2.12(0.95)$ & 3.28 & .071 \\
Fácil ligar & $1.57(1.33)$ & $1.64(0.91)$ & $1.49(1.69)$ & 1.18 & .277 \\
\hline
\end{tabular}

Finalmente, al ser preguntados acerca de su interés por ser científico o científica, se advierte un interés medio alto tanto en chicos como en chicas (más del 70\%, si sumamos la categoría sí y quizás; solamente un 20\% expresa no tener ningún interés en ser científico/a - $19 \%$ para chicos y $22 \%$ para chicas-. Aunque la tendencia es ligeramente superior para los niños, se verifica que no hay asociación entre ser mujer u hombre y querer ser científico/a $\left(X^{2}=0.72\right.$; $\mathrm{p}=.869)($ ver tabla 6$)$.

Tabla 6. ¿Te gustaría ser cientifico/a? (frecuencias y porcentajes)

\begin{tabular}{lccccc}
\hline & Total N $(\%)$ & Niños n $(\%)$ & Niñas n $(\%)$ & $X^{2}$ & Sig \\
\hline Si & $134(35.4)$ & $72(36)$ & $62(34.8)$ & 0.72 & .869 \\
Quizás & $134(35.4)$ & $73(36.5)$ & $61(34.3)$ & & \\
No & $78(20.6)$ & $38(19)$ & $40(22.5)$ & & \\
No sabe & $32(8.5)$ & $17(8.5)$ & $15(8.4)$ & & \\
\hline
\end{tabular}

\section{Discusión}

A modo de síntesis podemos agrupar la discusión de los datos obtenidos en tres bloques relativos a los conocimientos, a las actitudes e imagen de la ciencia de las y los estudiantes de educación primaria, y a la motivación profesional de ser científico/a.

El nivel de conocimientos registrado sobre las aportaciones de la ciencia y los científicos/as, es medio; la presencia de respuestas erróneas justifica, una vez más, la necesidad de perfeccionar la alfabetización científica de los futuros ciudadanos/as. Además, no se puede concluir que sean los niños los que presentan mayores cotas de saber frente a las niñas; las diferencias identificadas, como hemos visto, son escasas y no manifiestan una tendencia clara. El estudio 
de Pérez (2012) es coincidente para los estudiantes de primaria y para los de secundaria indica que se incrementan ligeramente los porcentajes y se acentúan las diferencias entre chicos y chicas. Por el contrario, Nortes y Pro (2010) concluyen que los estudiantes de 11-12 años presentan un elevado conocimiento general de las aportaciones de la ciencia y los/as científicos, con ciertas diferencias significativas de género, mostrando los niños un mayor conocimiento en cuanto a las aportaciones de la ciencia.

El otro pilar de esta investigación atiende a las actitudes de los niños y niñas hacia la ciencia, al valor que le conceden. Los resultados obtenidos parecen indicar una visión positiva de la ciencia, atribuyendo cualidades tales como interés, utilidad, mejora... así como la consideración de que sus aportaciones son más positivas que negativas. Asimismo, también presentan una buena imagen de los científicos y las científicas otorgándoles cualidades tales como entusiasmo, amistad, ... Además, los datos analizados asoman algunas diferencias en las opiniones hacia la ciencia de niños y niñas, con una orientación más positiva, más optimista y de mayor confianza en los niños que en las niñas; aunque en la mayoría de las atribuciones se constata uniformidad entre ambos grupos. También, en nuestro análisis hemos observado diferencias significativas en relación con el género que ocurren únicamente en algunas atribuciones de las y los científicos. La literatura consultada coincide fundamentalmente con lo apuntado para este estudio. Por una parte, concuerda en que la imagen que tienen los estudiantes de la ciencia es positiva (Delgado, Vallés y López 2014), aunque peor si se compara con otros países (Vázquez y Manassero 2007). En relación con las diferencias en actitudes en niños y niñas, los estudios consultados no marcan una evidencia clara en relación con la presencia de actitudes estereotipadas; algunos estudios (e.g. Nortes y Pro 2010, Pérez 2012, Schibeci, 1984) indican que no registran ningún efecto de género o efectos significativamente pequeños entre niños y niñas; por el contrario, otros estudios (Christidou et al. 2016, Jones et al. 2000, Kerr y Murphy 2012) alertan de los efectos significativos del género en las actitudes de los estudiantes hacia la ciencia.

Por último, la pertenencia al género masculino o femenino, en esta etapa, tampoco parece indicar que modifique las motivaciones profesionales de ser científico/a. Los resultados concuerdan con los apuntados en el estudio sobre las opiniones de los estudiantes de las clases de ciencias desde el último curso de primaria hasta finalizar secundaria de Marbà-Tallada y Márquez (2010); señalando una percepción más positiva en niños que en niñas de sexto de primaria por ser científico/a o tecnólogo/a, aunque, como ellas indican, la incidencia de género es menor de la esperada. Resultados semejantes han sido apuntados por Vázquez y Manassero (2011), los cuales concluyen que tanto para chicos como para chicas se produce un descenso de primaria a secundaria en el deseo de ser científicos o científicas, aunque confirman diferencias entre ambos en el perfil de descenso. El deseo de ser científicos/as muestra un perfil de descenso abrupto en secundaria para los chicos que mejora en segundo curso de secundaria; mientras las chicas, presentan un descenso regular, lo que, según los autores, puede indicar la raíz del rechazo de las mujeres hacia estudios superiores relacionados con la ciencia y la tecnología.

\section{Conclusiones}

La presente investigación se ha centrado en tres objetivos concretos. Respondiendo al primero de ellos, los resultados encontrados en esta investigación siguen justificando la necesidad de mejorar los conocimientos de los estudiantes sobre las aportaciones de la ciencia y los científicos/as, conocimientos que probablemente condicionen la imagen que el alumnado tenga de ellos/as. 
En cuanto a las actitudes del alumnado de educación primaria sobre las aportaciones de la ciencia y los/as científicos, los datos analizados indican una visión positiva del alumnado, y aunque en las mayoría de las atribuciones de la ciencia y los científicos/as no se aprecian diferencias en relación al género, si asoman diferencias en las opiniones hacia la ciencia, con una orientación más positiva en los niños que en las niñas.

Por último, señalar que las proyecciones profesionales del alumnado de primaria analizados en ser científicos o científicas no están asociadas al género.

En síntesis, podemos concluir que, el estudio efectuado, puede ser de utilidad para la práctica educativa al dar a conocer las actitudes de los estudiantes hacia la ciencia desde una perspectiva de género que debe ser tenida en cuenta en las intervenciones docentes. Si bien, se puede derivar un mensaje alentador de los resultados obtenidos, ya que parece que se atribuyen actitudes más sexistas hacia la ciencia de las que realmente se confirman en estas edades; habrá que seguir analizando la evolución de las mismas a lo largo del tiempo para identificar la trayectoria seguida y en qué momento aparecen. Porque efectivamente siguen manifestándose en la sociedad actual diferencias en la elección de estudios, escasa presencia de la mujer en cargos, en reconocimientos (49 mujeres con premio Nobel frente a 844 hombres), así como en la proyección de las mujeres investigadoras. Según datos que recoge el informe Científicas en Cifras 2017 (Puy 2018) el número de mujeres que obtienen el doctorado en España, primer paso para iniciar una carrera académica científica, se iguala al de los hombres, sin embargo la brecha sigue existiendo en los órganos de gobierno de universidades y OPIs (Organismos Públicos de Investigación).

Finalmente, en relación a las limitaciones de este estudio podemos destacar las siguientes. En primer lugar, el tamaño y distribución de la muestra que, aun siendo un " $\mathrm{n}$ " considerable, éste podría ampliarse y aumentar el ámbito geográfico, pudiendo llegar a toda la comunidad autónoma de Galicia. Otra limitación, que plantea la oportunidad de una futura investigación, sería completar la investigación actual con una de tipo cualitativo, que permitiera aproximarnos a las causas y a la comprensión de los datos cuantitativos, incluso que diera vOz también a los y las docentes implicados. Y, finalmente, otra limitación tiene que ver con el diseño adoptado; al tratarse de un diseño transversal la información se registra en un único momento temporal -cuando los estudiantes cursan sexto de educación primaria- lo que no permite visibilizar en qué momento se producen los cambios ni cuál va a ser su evolución (que podría completarse con un estudio longitudinal).

La utilización de instrumentos como los utilizados en la presente investigación permiten al profesorado detectar, en edades tempranas y desde una perspectiva de género, posibles diferencias relacionadas con el conocimiento y actitudes hacia la ciencia así como sus proyecciones hacia profesiones "científicas". La ciencia está presente en casi todas las acciones cotidianas del ser humano, particularmente en el mundo occidental; a pesar de ello, resulta llamativa la limitada formación científica que presentan la ciudadanía media. La alfabetización científica del alumnado, mejorar su actitud hacia la ciencia y los científicos/as, así como vencer las barreras de género deberían ser objetivos básicos de la enseñanza de las ciencias en la escuela primaria.

\section{Referencias}

Arandia E., Zuza K., Guisasola J. (2016) Actitudes y motivaciones de los estudiantes de ciencias en Bachillerato y Universidad hacia el aprendizaje de la Física. Revista Eureka sobre Enseñanza y Divulgación de las Ciencias, 13 (3), 558-573. 
Archer L., DeWitt J., Osborne J., Dillon J., Willis B., Wong B. (2010) "Doing” science versus "being" a scientist: Examining 10/11-year-old schoolchildren's constructions of science through the lens of identity. Science Education, 94 (4), 617-639. doi: $10.1002 /$ sce.20399

Bamberger Y. M. (2014) Encouraging Girls into Science and Technology with Feminine Role Model: Does This Work? Journal of Science Education and Technology, 23 (4), 549-561. doi: 10.1007/s10956-014-9487-7

Chachashvili-Bolotin S., Milner-Bolotin M., Lissitsa S. (2016) Examination of factors predicting secondary students' interest in tertiary STEM education. International Journal of Science Education, 38 (3), 366-390.

Christidou V., Bonoti F., Kontopoulou A. (2016) American and Greek Children's Visual Images of Scientists. Science \& Education, 25 (5-6), 497-522.

Dawson, C. (2000) Upper primary boys' and girls' interests in science: Have they changed since 1980? International Journal of Science Education, 22, 557-570.

Delgado J., Vallés C. López M. A. (2014, septiembre) Las actitudes hacia la ciencia de alumnos de Educación Primaria: una primera aproximación. XXVI Encuentros de Didáctica de las Ciencias Experimentales. Huelva, España.

Fernández C. M., Torío S., García O. (2015) Hombres y mujeres ante su formación universitaria. Expectativas de éxito en estudios científicos-tecnológicos y barreras percibidas. Investigación en la Escuela, 87, 21-33.

Gago J. M., Ziman J., Caro P., Constantinou C., Davies G., Parchmannn I., ... Sjøberg S. (2004) Europe needs more scientists: report by the high level group on increasing human resources for science and technology in Europe. Recuperado de http://ec.europa.eu/research/conferences/2004/sciprof/pdf/final_en.pdf.

Gil-Flores J. (2012) Actitudes del alumnado español hacia las ciencias en la evaluación Pisa 2006. Enseñanza de las Ciencias, 30 (2), 131-152.

Gil-Pérez D., Vilches A. (2006) Educación ciudadana y alfabetización científica: mitos y realidades. Revista Iberoamericana de Educación, 42, 31-53.

Haladyna T., Shaughnessy J. (1982) Attitudes towards science: A quantitative synthesis. Science Education, 66, 547-563. doi: 10.1002/sce.3730660406

Hill C.; Corbett C., Rose A. (2010) Why So Few? Women in Science, Technology, Engeneering and Mathematics. Washington, DC: AAUW. Recuperado de http:// files.eric.ed.gov/ fulltext/ED509653.pdf

Holmegaard, H. T., Madsen L. M., Ulriksen, L. (2012) To choose or not to choose science: constructions of desirable identities among young people considering a STEM higher education programme. International Journal of Science Education, 36 (2), 186-215.

Jones M. G., Howe A., Rua M. J. (2000) Gender differences in students' experiences, interests, and attitudes toward science and scientists. Science Education, 84 (2), 180-192.

Kahraman N., Sungur-Vural S. (2014) The contribution of gender, socio-economic status and socio-cultural influence to Turkish students' task value beliefs in science. Research in Education, 91 (1), 30-44.

Kerr K., Murphy C. (2012) Children's attitudes to primary science. En B. Fraser, K. Tobin \& C. J. McRobbie (Eds.), Second international handbook of science education (pp. 627-649). Springer Netherlands. 
Khanyane M., Mokuku T., Nthathakane M. C. (2016) Perceived gender differences in performance in science: the case of Lesotho secondary schools. African Journal of Research in Mathematics, Science and Technology Education, 20 (3), 278-288.

Marbà-Tallada A., Márquez, C. (2010) ¿Qué opinan los estudiantes de las clases de ciencias? Un estudio transversal de sexto de primaria a cuarto de ESO. Enseñanza de las Ciencias, 28 (1), 19-30.

Murphy C., Beggs J. (2003) Children's perceptions of school science. School Science Review, 84 (308), 109-116.

Murphy C., Beggs J., Carlisle K., Greenwood J. (2004) Students as catalysts in the classroom: The impact of co-teaching between science student teachers and primary classroom teachers on children's enjoyment and learning of science. International Journal of Science Education, 26, 1023-1035.

Nortes, R., Pro, A. (2010) Actitudes hacia las ciencias de los alumnos de Educación primaria de la región de Murcia. Comunicación presentada en II Jornadas del máster en investigación e innovación en Educación infantily Educación primaria. Murcia.

Nosek, B. A., Smyth, F. L., Sriram, N., Lindner, N. M., Devos T., Ayala A., ... Kesebir S. (2009) National differences in gender-science stereotypes predict national sex differences in science and math achievement. Proceedings of the National Academy of Sciences, 106 (26), 10593-10597.

OECD (Organisation for Economic Cooperation and Development) (2013) PISA 2015 Draft Science Framework. OECD.

Osborne J., Simon S., Collins S. (2003) Attitudes towards science: A review of the literature and its implications. International Journal of Science Education, 25 (9), 1049-1079.

Patall E. A., Steingut R. R., Freeman J. L., Pituch K. A., Vázquez A. C. (2018) Gender disparities in students'motivational experiences in high school science classromms. Science Education, 102 (5), 951-977.

Pérez A. (2012) Actitudes hacia la Ciencia en Primaria y Secundaria (Tesis Doctoral). Universidad de Murcia, España. http://www.tesisenred.net/handle/10803/120484

Pérez A., Pro A. (2005) Evaluación nacional de actitudes y valores hacia la ciencia en entornos educativos. Madrid: FECYT.

Pro A., Pérez A. (2014) Actitudes de los alumnos de Primaria y Secundaria ante la visión dicotómica de la Ciencia. Enseñanza de las Ciencias, 32.3, 111-132.

Pro A., Tárraga P., Pérez A. (2009) ¿Científico? Sí, pero... Opinión de los escolares españoles sobre los científicos y su trabajo. Enseñanza de las Ciencias, 3649-3656.

Puy A. (Coord) (2016) Cientificas en cifras 2017. Estadísticas e indicadores de la (des)igualdad de género en la formación y profesión científica. Madrid: Ministerio de Ciencia, Innovación y Universidades. https://icono.fecyt.es/informes-y-publicaciones/cientificas-en-cifras

Rossi A. E., Barajas M. (2015) Elección de estudios CTIM y desequilibrios de género. Enseñanza de las Ciencias, 33 (3), 59-76.

Schreiner C., Sjøberg S. (2004) Relevance of science education: Sowing the Seeds of ROSE. Oslo: Acta Didactica

Schibeci R. A. (1984) Attitudes to science: An update. Studies in Science Education, 11, 26-59. 
Selim M. A., Shrigley R. L. (1983) The group-dynamics approach: A socio-psychological approach for testing the effect of discovery and expository teaching on the science achievement and attitude of young Egyptian students. Journal of Research in Science Teaching, 20, 213-224. doi: 10.1002/tea.3660200305

Simpson A., Che S. M., Bridges W. C. (2016) Girls' and Boys' Academic Self-Concept in Science in Single-Sex and Coeducational Classes. International Journal of Science and Mathematics Education, 14 (8), 1407-1418.

Tytler R., Osborne J. (2012) Student attitudes and aspirations towards science. En B. Fraser, K. Tobin \& C. J. McRobbie (Eds.), Second international handbook of science education (pp. 597625). Springer: Netherlands.

Vázquez A., Manassero M. A. (2007) En defensa de las actitudes y emociones en la educación científica (II): Evidencias empíricas derivadas de la investigación. Revista Eureka sobre Enseñanza y Divulgación de las Ciencias, 4 (3), 417-441.

Vázquez A., Manassero M. A. (2011) El descenso de las actitudes hacia la ciencia de chicos y chicas en la educación obligatoria. Ciencia \& Educaçao, 17 (2), 249-268.

Vázquez A., Manassero M. A. (2015) La elección de estudios superiores científico-técnicos: análisis de algunos factores determinantes en seis países. Revista Eureka sobre Enseñanza y Divulgación de las Ciencias, 12 (2), 264-277.

Wang M. T., Degol J. L. (2017) Gender gap in science, techonology, engineering and mathematics (STEM): Current knowledge, implications for practice, policy and future directions. Educational Psichology Review, 29 (1), 119-140.

Watts R. (2014) Females in science: a contradictory concept? Educational Research, 56 (2), 126136. 


\section{Anexo 1}

\section{DATOS DE IDENTIFICACIÓN}

Edad: Sexo:

Centro:

Localidad:

Provincia:

\section{APORTACIONES DE LA CIENCIA Y LOS CIENTÍFICOS}

1. Hay muchos objetos que se deben a descubrimientos de la ciencia. Entre los que aparecen a continuación señala con un " $\underline{S}$ " los que se deben fundamentalmente a los científicos; con un " $\underline{N o}$ " los que no se deben a ellos; con un "En parte" cuando los científicos han participado, pero no han sido los únicos; y con "No lo se" cuando no lo tengas muy claro.

\begin{tabular}{|l|l|l|l|l|}
\hline & Sí & No & En parte & No lo sé \\
\hline Cuadro de un pintor & & & & \\
\hline Árboles & & & & \\
\hline Rueda & & & & \\
\hline Pan & & & & \\
\hline Moda & & & & \\
\hline Teléfonos & & & & \\
\hline Bombas & & & & \\
\hline Bicicletas & & & & \\
\hline
\end{tabular}

2. De las cosas que vienen a continuación señala con un " $\underline{\underline{P}}$ " las que crees que se deben a los científicos; con un " $N o$ " las que no se deben a ellos; con un "Sí, pero sólo en parte" aquellas en las que piensas que los científicos han participado, aunque también lo hayan hecho otras personas que no son científicos; y con "No lo sé" cuando no lo tengas claro.

\begin{tabular}{|c|c|c|c|c|}
\hline & Sí & No & $\begin{array}{l}\text { Sí, pero solo } \\
\text { en parte }\end{array}$ & No lo sé \\
\hline \multicolumn{5}{|c|}{ La Constitución española } \\
\hline \multicolumn{5}{|c|}{ Las reglas del fútbol } \\
\hline \multicolumn{5}{|c|}{ Las series de televisión } \\
\hline \multicolumn{5}{|c|}{ Las vacunas } \\
\hline \multicolumn{5}{|c|}{ Los horóscopos que predicen el futuro } \\
\hline \multicolumn{5}{|c|}{ El Quijote } \\
\hline \multicolumn{5}{|c|}{ La Revolución Industrial } \\
\hline \multicolumn{5}{|c|}{ Los viajes espaciales } \\
\hline \multicolumn{5}{|c|}{ Los coches de Fórmula 1} \\
\hline \multicolumn{5}{|c|}{ Un huevo frito } \\
\hline \multicolumn{5}{|c|}{ La música } \\
\hline El fuego & & & & \\
\hline
\end{tabular}


3. Mucha gente ha opinado sobre la ciencia y queremos saber tu opinión. Por favor, di tu grado de acuerdo con las frases que aparecen:

\begin{tabular}{|l|l|l|l|l|}
\hline & $\begin{array}{c}\text { Nada de } \\
\text { acuerdo }\end{array}$ & $\begin{array}{c}\text { Poco de } \\
\text { acuerdo }\end{array}$ & $\begin{array}{c}\text { Bastante de } \\
\text { acuerdo }\end{array}$ & $\begin{array}{c}\text { Totalmente } \\
\text { de acuerdo }\end{array}$ \\
\hline La curiosidad es esencial en la ciencia & & & & \\
\hline La ciencia debe ser eliminada de la escuela & & & & \\
\hline $\begin{array}{l}\text { La ciencia no tiene mucho interés para la gente que no es } \\
\text { científica }\end{array}$ & & & & \\
\hline Un futuro mejor depende de la ciencia & & & & \\
\hline La ciencia es completamente aburrida & & & & \\
\hline La ciencia es tremendamente útil & & & & \\
\hline $\begin{array}{l}\text { La ciencia nos defiende de quien pretende engañarnos } \\
\text { (adivinos, brujas,...) }\end{array}$ & & & & \\
\hline
\end{tabular}

\section{MIS OPINIONES SOBRE CIENCIA Y TECNOLOGÍA}

¿En qué medida estás de acuerdo con las siguientes afirmaciones? (Selecciona una de las opciones. Si no entiendes, no marques ninguna opción)

\begin{tabular}{|c|c|c|c|c|}
\hline & $\begin{array}{c}\text { Muy en } \\
\text { desacuerdo }\end{array}$ & Desacuerdo & Acuerdo & $\begin{array}{l}\text { Muy de } \\
\text { acuerdo }\end{array}$ \\
\hline \multicolumn{5}{|l|}{ La ciencia y la tecnología son importantes para la sociedad } \\
\hline \multicolumn{5}{|l|}{$\begin{array}{l}\text { La ciencia y la tecnología encontraron solución a } \\
\text { enfermedades como VIH/SIDA, cáncer,... }\end{array}$} \\
\hline \multicolumn{5}{|l|}{$\begin{array}{l}\text { Gracias a la ciencia y la tecnología habrá mayores } \\
\text { oportunidades para generaciones futuras }\end{array}$} \\
\hline \multicolumn{5}{|l|}{$\begin{array}{l}\text { La ciencia y la tecnología hacen nuestra vida más saludable, } \\
\text { fácil y cómoda }\end{array}$} \\
\hline \multicolumn{5}{|l|}{ Las nuevas tecnologías hacen el trabajo más interesante } \\
\hline \multicolumn{5}{|l|}{$\begin{array}{l}\text { Los beneficios de la ciencia son más importantes que los } \\
\text { perjuicios que causan }\end{array}$} \\
\hline \multicolumn{5}{|l|}{$\begin{array}{l}\text { La ciencia y la tecnología ayudan a erradicar la pobreza y el } \\
\text { hambre en el mundo }\end{array}$} \\
\hline \multicolumn{5}{|l|}{$\begin{array}{l}\text { La ciencia y la tecnología pueden resolver casi todos los } \\
\text { problemas }\end{array}$} \\
\hline \multicolumn{5}{|l|}{ La ciencia y la tecnología ayudan a los pobres } \\
\hline \multicolumn{5}{|l|}{$\begin{array}{l}\text { La ciencia y la tecnología son la causa de los problemas } \\
\text { ambientales }\end{array}$} \\
\hline \multicolumn{5}{|l|}{ Un país desarrollado necesita de la ciencia y la tecnología } \\
\hline \multicolumn{5}{|l|}{$\begin{array}{l}\text { La ciencia y la tecnología benefician principalmente a los } \\
\text { países desarrollados }\end{array}$} \\
\hline \multicolumn{5}{|l|}{$\begin{array}{l}\text { Los científicos siguen el método científico, que siempre los } \\
\text { llevan a respuestas concretas }\end{array}$} \\
\hline \multicolumn{5}{|l|}{ Hemos de confiar siempre en lo que dicen los científicos/as } \\
\hline \multicolumn{5}{|l|}{ Las personas científicas son neutrales y objetivas } \\
\hline $\begin{array}{l}\text { Las teorías científicas se desarrollan y cambian } \\
\text { constantemente }\end{array}$ & & & & \\
\hline
\end{tabular}

